\title{
Peripheral arterial tonometry as a method of measuring reactive hyperaemia correlates with organ dysfunction and prognosis in the critically ill patient: a prospective observational study
}

\author{
Luis Filipe Malheiro ${ }^{1,2,3,7} \mathbb{D} \cdot$ Rita Gaio $^{4}\left(\mathbb{D} \cdot\right.$ Manuel Vaz da Silva $^{5}$ (D) Sandra Martins ${ }^{6}\left(\mathbb{D} \cdot\right.$ António Sarmento $^{1,2,3} \mathbb{( D}$. \\ Lurdes Santos ${ }^{1,2,3}$ (D)
}

Received: 2 June 2020 / Accepted: 24 August 2020 / Published online: 5 September 2020

(c) Springer Nature B.V. 2020

\begin{abstract}
Predictions of mortality may help in the selection of patients who benefit from intensive care. Endothelial dysfunction is partially responsible for many of the organic dysfunctions in critical illness. Reactive hyperaemia is a vascular response of the endothelium that can be measured by peripheral arterial tonometry (RH-PAT). We aimed to assess if reactive hyperaemia is affected by critical illness and if it correlates with outcomes. Prospective study with a cohort of consecutive patients admitted to an Intensive Care Unit. RH-PAT was accessed on admission and on the 7th day after admission. Early and late survivors were compared to non-survivors. The effect of RH-PAT variation on late mortality was studied by a logistic regression model. The association between RH-PAT and severity scores and biomarkers of organic dysfunction was investigated by multivariate analysis. 86 patients were enrolled. Mean $\ln (\mathrm{RHI})$ on admission was 0.580 and was significantly lower in patients with higher severity scores $(\mathrm{p}<0.01)$ and early non-survivors $(0.388 ; \mathrm{p}=0.027)$. The model for prediction of early-mortality estimated that each 0.1 decrease in $\ln (\mathrm{RHI})$ increased the odds for mortality by $13 \%$. In 39 patients, a 2nd RH-PAT measurement was performed on the 7 th day. The variation of $\ln (\mathrm{RHI})$ was significantly different between non-survivors and survivors $(-24.2 \%$ vs. $63.9 \%, p=0.026)$. Ln(RHI) was significantly lower in patients with renal and cardiovascular dysfunction ( $<<0.01)$. RHPAT is correlated with disease severity and seems to be an independent marker of early mortality, cardiovascular and renal dysfunctions. RH-PAT variation predicts late mortality. There appears to be an RH-PAT impairment in the acute phase of severe diseases that may be reversible and associated with better outcomes.
\end{abstract}

Keywords Reactive hyperaemia $\cdot$ Critical illness $\cdot$ Intensive care $\cdot$ Prognosis $\cdot$ Endothelial dysfunction $\cdot$ Peripheral arterial tonometry $\cdot$ Organic dysfunction

Luis Filipe Malheiro

lmalha@gmail.com

1 Intensive Care Unit, Infectious Diseases Department, Centro Hospitalar de São João, Porto, Portugal

2 Institute for Innovation and Health Research (I3S), Institute of Biomedical Engineering (INEB), Nephrology and Infectious Diseases Research Group, University of Porto, Porto, Portugal

3 Department of Medicine Faculty of Medicine, University of Porto, Porto, Portugal

4 Department of Mathematics, Faculty of Science Sciences and CMUP, Centre of Mathematics of the University of Porto; University of Porto, Porto, Portugal
5 Department of Pharmacology and Therapeutics, Faculty of Medicine, University of Porto, Porto, Portugal

6 Clinical Pathology Department, Centro Hospitalar de São João and EPIUnit, Institute of Public Health, University of Porto, Porto, Portugal

7 Present Address: Serviço de Doenças Infeciosas, Centro Hospitalar de São João, Alameda Prof. Hernâni Monteiro, 4200-319 Porto, Portugal 


\section{Introduction}

Mortality in the Intensive Care Unit (ICU) is the highest among all hospital departments [1-3]. Correct predictions of mortality may help in the selection of patients who will benefit from intensive care and decide about treatment burden and success rates.

Endothelial cells are prone to damage and may become dysfunctional in acute and chronic diseases, in which inflammatory cytokines lead the endothelial cells to an activated phenotype [4]. In acute diseases such as sepsis, trauma and major surgery, acute endothelial dysfunction contributes to microvascular impairment, with decreases in nitric oxide (NO) production, whose manifestations include increased vascular permeability, dysregulated haemostasis, loss of vascular tone regulation and decreased oxygen uptake, culminating in multiorgan failure and increased mortality $[5,6]$. Despite its role in disease, the endothelium is rarely included in clinical algorithms for the assessment of disease severity in acute illnesses [7].

There are many methods by which endothelial function may be measured, each of them focusing on one of the many physiological roles of the endothelium. Reactive hyperaemia $(\mathrm{RH})$ is a flow-induced, endothelial-dependent vasodilatory vascular response, that can be non-invasively measured at the bedside and is partially dependent on NO [8]. Several tools, such as peripheral arterial tonometry (RH-PAT) and flow mediated dilation (FMD), have been validated for measuring RH [9-12]. RH-PAT is considered an accurate marker of endothelial dysfunction and an independent predictor of cardiovascular disease (CVD) and mortality in patients with chronic inflammatory diseases [13]. Recently, evidence that RH-PAT may correlate with disease severity and mortality, in patients with sepsis and ST-elevation myocardial infarction, suggests that RH-PAT may also be used for accessing prognosis in the critically ill patient [10, 14]. However, it is not known to what extent RH-PAT predicts organic dysfunction or mortality, if these changes in $\mathrm{RH}$ are also present in acute conditions in the critically ill patient other than sepsis and myocardial infarction, if the acute RH impairment is reversible, nor the prognostic implications of $\mathrm{RH}$ reversibility.

In this study, our main objective was to verify if there is an association between RH-PAT and outcomes in the critically ill patient. Furthermore, we questioned if RH-PAT and other endothelial biomarkers correlate with variables of organic dysfunction; if the acute impairment in the RHPAT is reversible and if this reversibility correlates with long-term outcomes.

\section{Methods}

\subsection{Study design}

We performed a prospective cohort study with consecutive patients admitted to an ICU in a tertiary care centre, in whom RH-PAT was measured on admission and patients were observed over a period of 12 months and grouped as survivors or non-survivors. Written consent was obtained from the patient if awake, or from a legal representative. Ethic approval for the study was obtained from the Hospitals' Ethics Committee.

\subsection{Participant selection and eligibility criteria}

Adult patients admitted to the ICU over a period of 12 months (September 2016 to September 2017) were evaluated for study eligibility in the first $24 \mathrm{~h}$ after admission. Patients were considered eligible if they fulfilled any of the following criteria: respiratory failure from any cause requiring invasive positive-pressure mechanical ventilatory support, patients requiring extracorporeal respiratory support, patients requiring continuous intravenous vasoactive drugs, patients requiring continuous intravenous medication to control seizures and supplementary oxygen/ airway monitoring and need of monitoring and support for two or more organ systems. Patients were not included based on the exclusion criteria described in Table 1.

\subsection{Participant characterization and definition of primary and secondary outcomes}

Participants underwent clinical and demographic data collection and routine blood sampling for biochemical analysis on admittance. Severity was defined according to the Sequential Organic Failure Assessment (SOFA) score, Acute Physiology and Chronic Health Evaluation II (APACHE-II) score and Simplified Acute Physiology Score II (SAPS-II). The patients were characterized as having circulatory shock according to the guidelines described in Table 2 [15-17]. Organic dysfunctions were defined using biochemical and clinical assessments and the cut-off values were determined using available guidelines (Table 2) [15, 18-20]. Primary outcomes were early and late mortality, defined as death by the 28 th day and 12 th month, respectively. Secondary outcomes were considered as the presence of organic dysfunctions defined in Table 2. 
Table 1 Exclusion criteria used for defining organic dysfunction

Critically ill patients with an acute condition, admitted in the Intensive Care Unit, whose evaluation was not performed in the first $24 \mathrm{~h}$

OR

Patients with clinical or anatomic conditions that prevent endothelial dysfunction evaluation, such as:

a) Absence of the 2nd or 3rd pair of fingers

b) Mean arterial pressure $<50 \mathrm{mmHg}$, already under vasopressor support, in whom compression of the brachial artery may compromise distal perfusion

c) Known brachial artery stenosis or severe reactive vasospasm in response to recent arterial catheterization

d) Arterial-venous fistulae in any arm

e) Permanent tremor or restlessness that compromises evaluation

f) Absence of informed consent

g) Platelets $<20.000 / \mu \mathrm{L}$ or activated partial thromboplastin time $\geq 70$ s or international normalized ratio $\geq 2.0$

Table 2 Definitions used for establishing organic dysfunction

\begin{tabular}{|c|c|}
\hline Sepsis $^{\mathrm{a}}$ & $\begin{array}{l}\text { Strongly suspected or confirmed infectious source on admission } \\
\text { AND } \\
\text { A change in baseline of the total SOFA (Sequential Organ Failure Assessment) score of } \geq 2 \text { points }\end{array}$ \\
\hline Septic shock ${ }^{\mathrm{a}}$ & $\begin{array}{l}\text { Persisting hypotension requiring vasopressors to maintain mean arterial pressure } \geq 65 \mathrm{mmHg} \text {; } \\
\text { OR } \\
\text { Serum lactate level }>2 \mathrm{mmol} / \mathrm{L} \text { despite adequate volume resuscitation; }\end{array}$ \\
\hline Cardiogenic shock ${ }^{\mathrm{b}}$ & $\begin{array}{l}\text { Compatible clinical situation; } \\
\text { AND } \\
\text { Systolic blood pressure }<90 \mathrm{mmHg} \text { with adequate volume; } \\
\text { AND } \\
\text { Clinical or laboratory signs of hypoperfusion; }\end{array}$ \\
\hline Hypovolemic shock ${ }^{\mathrm{b}}$ & $\begin{array}{l}\text { Evidence of fluid loss, in the absence of infection or heart failure; } \\
\text { AND } \\
\text { Systolic blood pressure }<90 \mathrm{mmHg} \text {; } \\
\text { OR } \\
\text { Serum lactate level }>2 \mathrm{mmol} / \mathrm{L} \text {; }\end{array}$ \\
\hline Cardiovascular dysfunction $^{\mathrm{a}}$ & $\begin{array}{l}\text { Systolic blood pressure }<90 \mathrm{mmHg} \text { or Mean arterial pressure }<65 \mathrm{mmHg} \text { or lactate }>2.0 \mathrm{mmol} / \mathrm{L} \text { (after initial } \\
\text { fluid challenge) }\end{array}$ \\
\hline Renal dysfunction $^{\mathrm{c}}$ & $\begin{array}{l}\text { Stage 1: SCr1.5-1.9 times baseline or } \geq 0.3 \mathrm{mg} / \mathrm{dl} \text { increase or urinary flow } \leq 0.5 \mathrm{ml} / \mathrm{kg} / \mathrm{h} \text { for } 6-12 \mathrm{~h} \text {; } \\
\text { Stage 2: SCr 2.0-2.9 times baseline or } \leq 0.5 \mathrm{ml} / \mathrm{kg} / \mathrm{h} \text { for } \geq 12 \mathrm{~h} \\
\text { Stage 3: SCr } 3.0 \text { times baseline or Increase in serum creatinine to } \geq 4.0 \mathrm{mg} / \mathrm{dl} \text { or Initiation of renal replace- } \\
\text { ment therapy or Decrease in eGFR to }<35 \mathrm{ml} / \mathrm{min} / 1.73 \mathrm{~m} 2 \text { or urinary flow } \leq 0.3 \mathrm{ml} / \mathrm{kg} / \mathrm{h} \text { for } \geq 24 \mathrm{~h} \text { or Anuria } \\
\text { for } \geq 12 \mathrm{~h} \text {; }\end{array}$ \\
\hline Hematologic dysfunction $^{\mathrm{a}}$ & Platelets $<150 \times 10^{3} / \mu 1$ or prolonged prothrombin time $\geq 2 \mathrm{~s}$ \\
\hline Hepatic dysfunction $^{\mathrm{a}}$ & Serum bilirubin $\geq 2 \mathrm{mg} / \mathrm{dl}$ \\
\hline Respiratory dysfunction ${ }^{\mathrm{d}}$ & $\begin{array}{l}\text { Impaired oxygenation of acute onset: } \\
\text { Moderate- } \mathrm{P}_{\mathrm{a}} \mathrm{O} 2 / \mathrm{FiO} 2 \text { ratio } 100-200 \\
\text { Severe }-\mathrm{P}_{\mathrm{a}} \mathrm{O} 2 / \mathrm{FiO} 2 \text { ratio }<100\end{array}$ \\
\hline Neurological dysfunction ${ }^{\mathrm{a}}$ & Decrease in 1 point from basal GCS \\
\hline
\end{tabular}

$e G F R$ Estimated glomerular filtration rate, $\mathrm{FiO} 2$ fraction of inspired oxygen, $\mathrm{P}_{a} \mathrm{O} 2$ partial arterial oxygen pressure, $\mathrm{SCr}$ Serum Creatinine

${ }^{\text {a }}$ Defined according to the SEPSIS-3 definitions ${ }^{15}$

${ }^{b}$ Defined according to the Consensus on circulatory shock and hemodynamic monitoring ${ }^{17}$

${ }^{\mathrm{c}}$ Defined according to the KDIGO guidelines on Acute Kidney Injury ${ }^{20}$

${ }^{\mathrm{d}}$ Defined according to the Acute respiratory distress syndrome: the Berlin Definition ${ }^{19}$

\subsection{Measuring vascular reactivity}

Reactive hyperaemia was accessed on admittance with the Endo-PAT 2000® tonometer (Itamar Medical, Cesarea, Israel). The evaluation was performed after, at least, $1 \mathrm{~h}$ of hemodynamic stability (defined as absence of vasopressor drugs dose adjustments or need for fluid bolus) in a controlled environment (room temperature $21.5^{\circ} \mathrm{C}$ ). For each assessment, a software profile was introduced, controlling for age, weight, estimated height, heart rate and arterial 
pressure. RH-PAT protocol was performed as suggested by the manufacturer and, with the patient in the dorsal recumbent position, a probe was introduced in each 2 nd finger and plethysmography was used to register the pulse wave amplitude (PWA) during the basal, occlusion and post-occlusion periods [21]. Using the Endo-PAT 2000 software (version 3.1.2) provided by the manufacturer, the RH index (RHI) was obtained by quantifying a ratio between the PWA from the pre- and post- occlusion periods. An example and explanation of RH-PAT in a healthy patient can be found in Fig. 1. Although official reference values for RHI are unavailable, RHI is considered to be a marker of endothelial dysfunction and mortality if under $1.68(\ln (\mathrm{RHI}) 0.51)$, and is generally regarded by the manufacturer as impaired if under $2.0(\ln (\mathrm{RHI})$ $0.69)$, whereas higher RHI values are considered normal endothelial responses [22].

\subsection{Evaluating patient progression}

Patients who survived and were not discharged from the ICU, were reassessed for RH-PAT on the 7th day after admittance. Patients who were discharged to other nonICU wards were excluded as the environmental variables (i.e. room temperature, humidity, luminosity, distractions) could not be controlled.

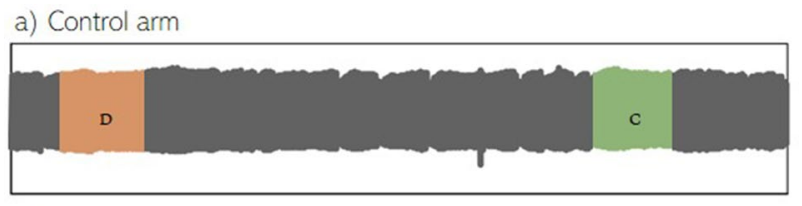

b) Occluded arm

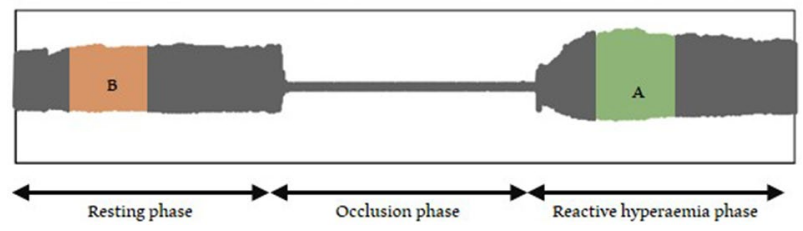

Fig. 1 Example of RH-PAT analysis in a healthy patient. The study starts by evaluating the basal PWA on both arms for $5 \mathrm{~min}$. This is followed by a sphygmomanometer cuff inflation in the 2nd portion of one of the arms, compressing the brachial artery flow. After a 5-min occlusion period, the cuff is deflated and a new evaluation of the PWA is carried for another $5 \mathrm{~min}$. After data registration, the manufacturer software analyses the results and computes a Reactive Hyperaemia Index (RHI). The RHI is obtained by calculating the ratio of the occluded arm's mean PWA 90-150 s post occlusion (a) to the mean basal PWA of the same arm (b). The result is divided by the same ratio from the control arm (C/D) to consider systemic vascular changes during the test. The final ratio is further multiplied by a baseline correction factor (Itamar Medical, Cesarea, Israel)

\subsection{Immunologic evaluation}

Quantification of Syndecan-1, Endocan, and soluble E-selectin (sE-selectin) levels in plasma samples was performed by solid phase sandwich immunoassays using fluorescently dyed micron-sized polystyrene microspheres in the Human Premixed Multiplex kit (Magnetic Luminex ${ }^{\circledR}$ Assay, Cat. No. LXSAHM-04, kit lot No. L126978, R\&D Systems $®$, Inc.). Results were analysed with the Luminex 200TM xMAPTM Technology (Luminex Corp.) and the Luminex xPONENT ® Software (version 3.1).

\subsection{Statistical analysis}

Descriptive statistics consisted of mean and standard deviation (SD) for continuous variables, and absolute/relative frequencies for categorical variables. The association between two continuous variables was assessed by the Pearson correlation coefficient. Statistical inferences were drawn from the application of the t-test, chi-squared or Fisher's exact test, Pearson's correlation test or ANOVA followed by Dunnett's multiple comparisons with the absent category, as appropriate.

Multivariate analysis included linear regression for continuous responses and logistic regression for binary outcomes; results are presented as B (coefficient), OR (odds ratio) and 95\% CI (confidence interval for the OR). Only significant variables ( $p$-value $<0.05$ ) were considered in the final regression analysis model. The variation of RHI over time was quantified using the quotient $\left(\ln (\mathrm{RHI})_{(1 \mathrm{st} \text { day })^{-}}\right.$ $\left.\ln (\mathrm{RHI})_{(7 \text { th day })}\right) / \ln (\mathrm{RHI})_{(1 \text { st day })} \mid$. To test whether the evolution of RHI through time influenced mortality after 12 months, we fitted a logistic regression model including the relative difference in RHI as an explanatory variable. Due to the reduction in sample size, the estimation method used the penalized likelihood proposed by Firth, in order to avoid first-order bias [23]. The statistical analyses were performed with the R language and software environment for statistical computation, version 2.3.3. Statistical significance was fixed at 0.05 .

\section{Results}

\subsection{Participants description}

From 115 patients admitted during a period of 12 months, 86 patients were enrolled, of which 40 patients were included in the follow-up evaluation. The workflow of inclusion and exclusion can be found in Fig. 2. Patient characteristics and outcomes are described in Table 2 . The study population has 
Fig. 2 Flowchart of study procedures. ICU Intensive care unit, $R H-P A T$ reactive hyperaemia-Peripheral Arterial Tonometry

115 patients admitted to the Intensive Care Unit

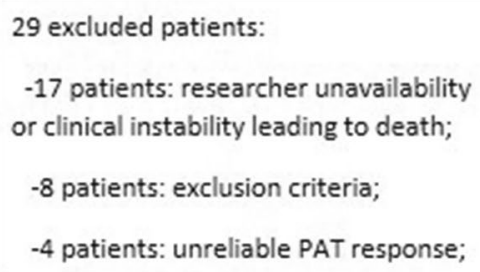

been previously enrolled in a study of endothelial dysfunction and sepsis and results from this study group have been reported [24].

\subsection{Endothelial dysfunction on admission and early mortality}

Early and late mortality were of $24.4 \%$ (21 patients) and $36.6 \%$ (31 patients), respectively. The clinical characteristics between both outcomes can be found in Table 3 . Non-survivors were significantly older (age $\geq 60$ years old) and presented more cardiovascular comorbidities (arterial hypertension, diabetes mellitus (DM), dyslipidaemia and coronary disease) and malignancies (metastatic solid neoplasia) than survivors. Non-survivors had significantly higher severity indexes (APACHEII and SAPSII) but did not have higher organ failure assessment scores (SOFA) or individual organic dysfunctions. RHI and plasma concentrations of Syndecan-1, sE-selectin and Endocan, presented skewed distributions and, therefore, analyses were performed on log-transformed data (ln).

Mean $\ln (\mathrm{RHI})$ on admission was 0.580 (SD 0.462, range -0.91 to 1.61 ) and was found to be significantly lower in patients with higher severity scores (APACHEII and SAPSII score, $p<0.01)$ and early non-survivors $(p=0.027)$ (Fig. 3d). As previously reported in this cohort, $\ln (\mathrm{RHI})$ is influenced, not only by the presence of multiple comorbidities such as dyslipidaemia, peripheral arterial disease and coronary disease, but also by disease severity such as the presence of shock and high severity scores [24]. Syndecan-1 levels were found to be significantly lower in early non-survivors $(\mathrm{p}=0.024)$ but not in late non-survivors. There was a tendency for lower $\ln (\mathrm{RHI})$ values in late non-survivors $(\mathrm{p}=0.095)$ (Fig. 3e). 
Table 3 Patient characteristics and univariate analysis of factors influencing early and late mortality

\begin{tabular}{|c|c|c|c|c|c|c|c|c|}
\hline & & \multirow{2}{*}{$\begin{array}{l}\text { Total } \\
(\mathrm{n}=86)\end{array}$} & \multicolumn{3}{|c|}{ Early mortality-28 days } & \multicolumn{3}{|c|}{ Late mortality -12 months } \\
\hline & & & $\begin{array}{l}\text { Survivors } \\
(\mathrm{n}=65)\end{array}$ & $\begin{array}{l}\text { Non-survivors } \\
(\mathrm{n}=21)\end{array}$ & p-value & $\begin{array}{l}\text { Survivors } \\
(\mathrm{n}=55)\end{array}$ & $\begin{array}{l}\text { Non-survivors } \\
(\mathrm{n}=31)\end{array}$ & P-value \\
\hline \multirow[t]{2}{*}{ Demographics } & Sex-male ${ }^{a}$ & $58(67.4 \%)$ & $44(67.7 \%)$ & $14(66.7 \%)$ & 0.931 & $34(61.8 \%)$ & $24(77.4 \%)$ & 0.138 \\
\hline & Age $\geq 60$ y.o ${ }^{a}$ & $35(40.7 \%)$ & $22(33.8 \%)$ & $13(61.9 \%)$ & 0.023 & $17(30.9 \%)$ & $18(58.1 \%)$ & 0.014 \\
\hline \multirow[t]{12}{*}{ Comorbidities } & $\mathrm{DM} 2^{\mathrm{a}}$ & $22(25.6 \%)$ & $12(18.5 \%)$ & $10(47.6 \%)$ & 0.008 & $11(20.0 \%)$ & $11(35.5 \%)$ & 0.114 \\
\hline & $\mathrm{AH}^{\mathrm{a}}$ & $43(50.0 \%)$ & $28(43.1 \%)$ & $15(71.4 \%)$ & 0.024 & $22(40 \%)$ & $21(67.7 \%)$ & 0.013 \\
\hline & Dyslipidaemia $^{a}$ & $35(40.7 \%)$ & $21(32.3 \%)$ & $14(66.7 \%)$ & 0.005 & $17(30.9 \%)$ & $18(58.1 \%)$ & 0.014 \\
\hline & Statin treatment ${ }^{\mathrm{a}}$ & $30(34.9 \%)$ & $19(29.2 \%)$ & $11(52.4 \%)$ & 0.053 & $15(27.3 \%)$ & $15(48.4 \%)$ & 0.049 \\
\hline & $\begin{array}{l}\text { Peripheral arterial } \\
\text { disease }^{\mathrm{a}}\end{array}$ & $21(24.4 \%)$ & $13(20.0 \%)$ & $8(38.1 \%)$ & 0.093 & $9(16.5 \%)$ & $12(38.7 \%)$ & 0.021 \\
\hline & $\mathrm{COPD}^{\mathrm{a}}$ & $13(15.1 \%)$ & $8(12.3 \%)$ & $5(23.8 \%)$ & 0.201 & $8(14.5 \%)$ & $5(16.1 \%)$ & 0.844 \\
\hline & $\begin{array}{l}\text { Auto-Immune } \\
\text { disease }^{\mathrm{a}}\end{array}$ & $5(4.8 \%)$ & $5(7.7 \%)$ & 0 & 0.190 & $4(7.3 \%)$ & $1(3.2 \%)$ & 0.441 \\
\hline & Smoking habits ${ }^{\mathrm{a}}$ & $28(32.6 \%)$ & $20(30.8 \%)$ & $8(38.1 \%)$ & 0.553 & $17(30.9 \%)$ & $11(35.5 \%)$ & 0.664 \\
\hline & Coronary disease $^{\mathrm{a}}$ & $24(27.9 \%)$ & $14(21.5 \%)$ & $10(47.6 \%)$ & 0.021 & $11(20.0 \%)$ & $13(41.9 \%)$ & 0.029 \\
\hline & $\begin{array}{l}\text { Haematological } \\
\text { cancer }^{\mathrm{a}}\end{array}$ & $2(2.3 \%)$ & $2(3.1 \%)$ & 0 & 0.416 & $1(1.8 \%)$ & $1(3.2 \%)$ & 0.678 \\
\hline & HIV $^{\mathrm{a}}$ & $12(14.0 \%)$ & $8(12.3 \%)$ & $4(14.0 \%)$ & 0.438 & $7(12.7 \%)$ & $5(16.1 \%)$ & $0.749 *$ \\
\hline & $\begin{array}{l}\text { Metastatic solid } \\
\text { neoplasia }^{a}\end{array}$ & $8(9.3 \%)$ & $4(6.2 \%)$ & $4(19.0 \%)$ & $0.095 *$ & $2(3.6 \%)$ & $6(19.4 \%)$ & $0.023^{*}$ \\
\hline \multirow[t]{15}{*}{ Severity } & APACHE score ${ }^{b}$ & $20.5(7.1)$ & $19.2(7.1)$ & $24.7(5.4)$ & 0.002 & $18.9(7.3)$ & $23.4(6.0)$ & 0.05 \\
\hline & SAPS2 Score ${ }^{b}$ & $55.4(20.0)$ & $52.9(20.6)$ & $63.8(16.5)$ & 0.049 & $51.85(20.4)$ & $52.6(17.9)$ & 0.03 \\
\hline & SOFA score ${ }^{\mathrm{b}}$ & $9.5(4.2)$ & $9.40(0.55)$ & $9.84(0.88)$ & 0.893 & $9.55(0.63)$ & $9.41(0.70)$ & 0.692 \\
\hline & $\begin{array}{l}\text { Cardiovascular } \\
\text { dysfunction }^{\mathrm{a}}\end{array}$ & $51(59.3 \%)$ & $37(56.9 \%)$ & $14(66.7 \%)$ & 0.429 & $32(58.2 \%)$ & $19(61.3 \%)$ & 0.778 \\
\hline & $\begin{array}{l}\text { Respiratory } \\
\text { dysfunction }^{\mathrm{a}}\end{array}$ & $43(50.0 \%)$ & $45(69.2 \%)$ & $16(76.2 \%)$ & 0.541 & $38(69.1 \%)$ & $23(74.2 \%)$ & 0.617 \\
\hline & Renal dysfunction $^{\mathrm{a}}$ & $19(22.1 \%)$ & $16(24.6 \%)$ & $9(42.9 \%)$ & 0.109 & $15(27.3 \%)$ & $10(32.3 \%)$ & 0.625 \\
\hline & $\begin{array}{l}\text { Neurological } \\
\text { dysfunction }^{\mathrm{a}}\end{array}$ & $46(53.5 \%)$ & $35(53.8 \%)$ & $13(61.9 \%)$ & 0.518 & $31(56.4 \%)$ & $17(54.8 \%)$ & 0.891 \\
\hline & $\begin{array}{l}\text { Haematological } \\
\text { dysfunction }\end{array}$ & $24(27.9 \%)$ & $41(63.1 \%)$ & $10(47.6 \%)$ & 0.210 & $37(67.3 \%)$ & $14(45.2 \%)$ & 0.045 \\
\hline & Hepatic dysfunction $^{a}$ & $23(26.7 \%)$ & $15(23.1 \%)$ & $6(28.6 \%)$ & 0.610 & $14(25.5 \%)$ & $7(22.6 \%)$ & 0.766 \\
\hline & Norepinephrine use ${ }^{\mathrm{a}}$ & $41(47.6 \%)$ & $31(47.5 \%)$ & $10(47.6 \%)$ & 0.995 & $27(49.1 \%)$ & $14(45.1 \%)$ & 0.726 \\
\hline & $\begin{array}{l}\text { Norepinephrine } \\
\text { dose }^{\mathrm{b}, \mathrm{d}}(\mathrm{mcg} / \mathrm{kg} / \text { minute) }\end{array}$ & $0.447(0.545)$ & $0.433(0.601)$ & $0.489(0.337)$ & 0.813 & $0.451(0.639)$ & $0.438(0.310)$ & 0.815 \\
\hline & Sepsis $^{\mathrm{a}}$ & $58(67.4 \%)$ & $44(67.7 \%)$ & $14(66.7 \%)$ & 0.931 & $38(69.1 \%)$ & $20(64.5 \%)$ & 0.664 \\
\hline & Shock $^{\mathrm{a}}$ & $37(43.0 \%)$ & $27(41.5 \%)$ & $10(47.6 \%)$ & 0.625 & $22(40.0 \%)$ & $15(48.4 \%)$ & 0.451 \\
\hline & Septic shock ${ }^{\mathrm{a}}$ & $29(33.7 \%)$ & $21(32.3 \%)$ & $8(38.1 \%)$ & 0.877 & $19(34.5 \%)$ & $10(32.3 \%)$ & 0.259 \\
\hline & Non-septic shock ${ }^{\mathrm{a}}$ & $8(9.3 \%)$ & $6(9.2 \%)$ & $2(9.5 \%)$ & & $3(5.5 \%)$ & $5(16.1 \%)$ & \\
\hline \multirow{4}{*}{$\begin{array}{l}\text { Endothelial evalu- } \\
\text { ation }\end{array}$} & $\ln (\mathrm{RHI})^{\mathrm{b}}$ & $0.580(0.462)$ & $0.642(0.389)$ & $0.388(0.607)$ & 0.027 & $0.643(0.365)$ & $0.469(0.587)$ & 0.095 \\
\hline & $\ln (\text { Endocan })^{\mathrm{b}}{ }_{(\mathrm{pg} / \mathrm{mL})}$ & $7.11(0.09)$ & $7.06(0.10)$ & $7.35(0.24)$ & 0.226 & $7.09(0.12)$ & $7.16(1.16)$ & 0.708 \\
\hline & $\begin{array}{l}\ln (\text { Syndecan-1 })^{\mathrm{b}} \\
(\mathrm{pg} / \mathrm{mL})\end{array}$ & $8.57(0.07)$ & $8.49(0.07)$ & $8.88(0.16)$ & 0.024 & $8.51(0.09)$ & $8.69(0.11)$ & 0.215 \\
\hline & $\begin{array}{l}\mathrm{Ln}(\mathrm{sE}-\text { selectin })^{\mathrm{b}} \\
\quad(\mathrm{pg} / \mathrm{mL})\end{array}$ & $10.66(0.10)$ & $10.48(0.11)$ & $10.55(0.56)$ & 0.576 & $10.70(0.12)$ & $10.60(0.19)$ & 0.643 \\
\hline
\end{tabular}

AH Arterial hypertension, APACHEII acute physiology and chronic health evaluation II, COPD chronic obstructive pulmonary disease, $D M 2$ diabetes mellitus II, HIV human immunodeficiency virus, Ln natural logarithm, RHI REACTIVE HYPERAEMIA INDEX, SAPSII simplified acute physiology score II, SOFA sequential organ failure assessment

${ }^{a}$ Categorical variables presented as absolute frequencies (percentages on column)

${ }^{\mathrm{b}}$ Continuous variables presented as mean (standard deviation); Means compared with students' t-test, frequencies compared using chi-squared ${ }^{\mathrm{c}}$ Fisher test 
Table 3 (continued)

${ }^{\mathrm{d}}$ Denominator is 41 patients using vasopressor

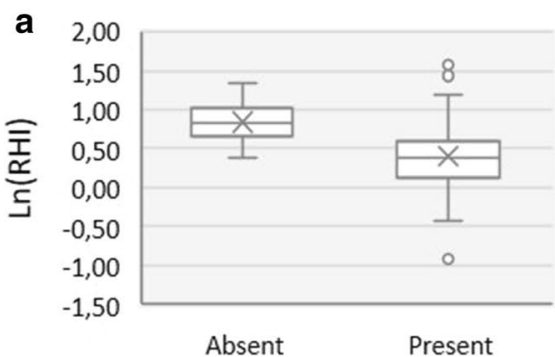

Cardiovascular dysfunction

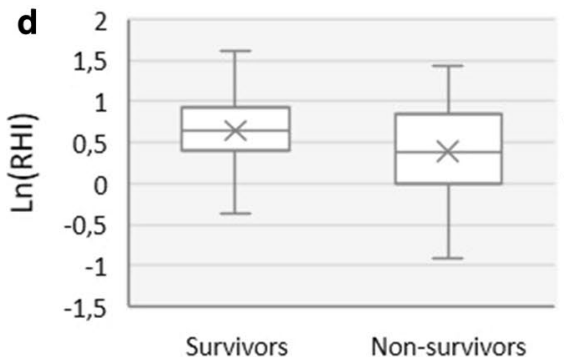

Early mortality

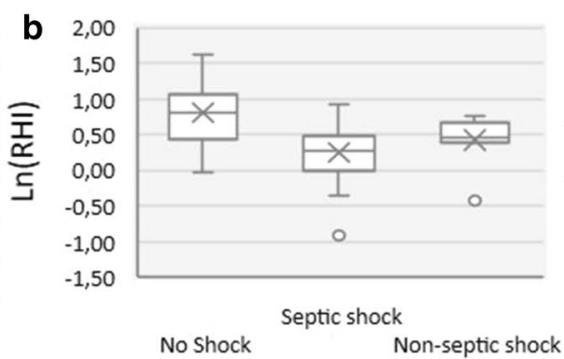

Circulatory shock

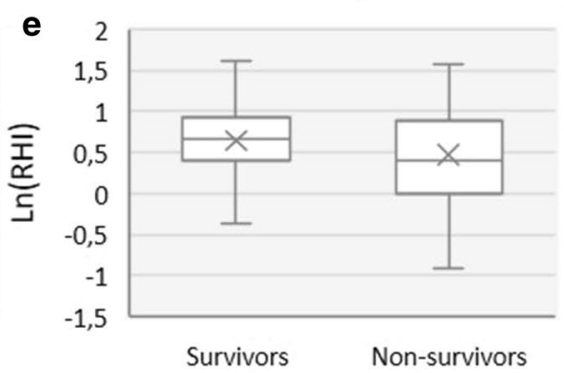

Late mortality

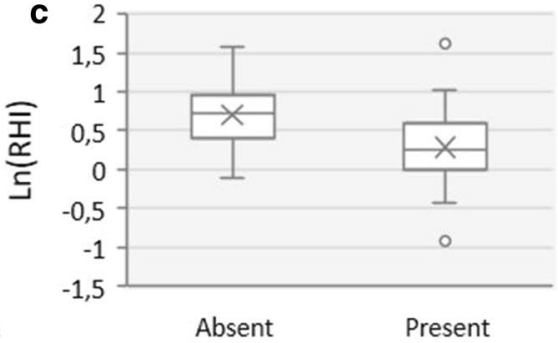

Renal dysfunction

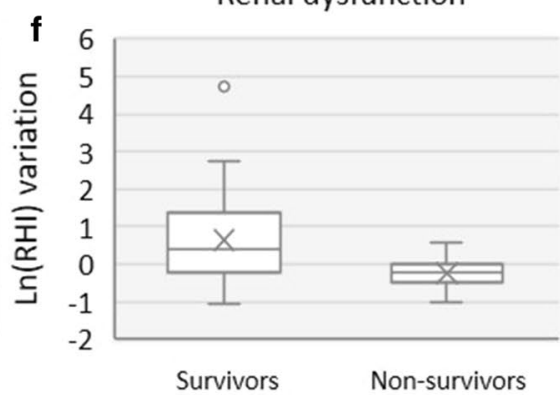

Late mortality
Fig. 3 Comparison of $\ln (\mathrm{RHI})$ with organic dysfunctions and mortality. a comparison of $\ln (\mathrm{RHI})$ on admission with cardiovascular dysfunction; b comparison of $\ln (\mathrm{RHI})$ on admission with circulatory shock dysfunction; c comparison of $\ln (\mathrm{RHI})$ on admission with renal dysfunction; $\mathbf{d}$ comparison of $\ln (\mathrm{RHI})$ on admission with early

The (crude) effect of $\ln (\mathrm{RHI})$ in early mortality was adjusted for all variables considered as significant in the univariate analysis. The best regression model evaluating the (adjusted) effect of $\ln (\mathrm{RHI})$ in early mortality, included $\ln (\mathrm{RHI})(\mathrm{B}=-1.222 ; \mathrm{OR}=0.295 ; \mathrm{p}=0.045 ; 95 \% \mathrm{CI}=0.089$ to 0.976$)$ and $\mathrm{DM}(\mathrm{B}=1.353 ; \mathrm{OR}=3.869 ; \mathrm{p}=0.015 ; 95 \%$ $\mathrm{CI}=1.299$ to 11.530 ) as explanatory variables. The model predicts that, at any given DM category, each 0.1 decrease in $\ln (\mathrm{RHI})$ increases the odds for mortality by $13 \%$ (1/ $\left.\mathrm{e}^{-1,222^{*} 0.1}=1.129\right)$. No other variables significantly improved the model, including the use or dose of norepinephrine.

\subsection{Endothelial dysfunction evolution and late mortality}

To evaluate to what extent the variation of $\ln (\mathrm{RHI})$ predicts late mortality, 40 patients with a 2 nd measurement of the $\ln (\mathrm{RHI})$ on the 7 th day were selected. One patient with necrotizing fasciitis was excluded from this group, as he improved after initial resuscitation and surgical control, but was later rendered inoperable and died after suspension of supportive measures. Characteristics of these 39 patients mortality (28th day); e comparison of $\ln (\mathrm{RHI})$ on admission with late mortality (12th month); $\mathbf{f}$ comparison of the variation of the $\ln (\mathrm{RHI})$ between admission and the 7th day with late mortality (12th month). In Natural logarithm, RHI reactive hyperaemia index

differed from the original ( $\mathrm{n}=86$ patients) as males were more prevalent $(76.9 \%$ vs $67.4 \%$ ), patients were younger (33.3\% vs $40.7 \%$ of patients older than 60 years old) and all comorbidities were less frequent. Severity measured by the APACHE-II, SAPS-II and SOFA scores did not differ between the two study samples.

The variation of $\ln (\mathrm{RHI})$ was significantly different between the $10(25.6 \%)$ non-survivors and the $29(74.4 \%)$ survivors (- 24.2\% (SD 44.9\%) vs $63.9 \%$ (SD 122\%), $\mathrm{p}=0.026$, respectively) (Fig. 3f). The corresponding logistic regression model predicted that patients in whom the $\ln (\mathrm{RHI})$ improved $\geq 10.7 \%$ were survivors and those with variations $<10.7 \%$ were non-survivors on the 12 th month after admission (overall correct classification $=66.7 \%$, sensibility $=90 \%$ and specificity $=58.6 \%$ ). Due to the limited number of cases, a multivariate analysis was not performed.

\subsection{Reactive hyperaemia on admission, organic dysfunction, and disease severity}

Comparison of $\ln (\mathrm{RHI})$ and other biomarkers of endothelial activation with organic dysfunctions can be found in Table 4 . 
Table 4 Analysis of endothelial dysfunction markers and organic dysfunctions

\begin{tabular}{|c|c|c|c|c|c|c|c|c|c|}
\hline \multirow{3}{*}{ Shock } & & \multicolumn{2}{|l|}{$\ln (\mathrm{RHI})$} & \multicolumn{2}{|c|}{$\ln (\text { Endocan })_{(\mathrm{pg} / \mathrm{mL})}$} & \multicolumn{2}{|c|}{$\mathrm{Ln}(\mathrm{sE}-$ Selectin $)(\mathrm{pg} / \mathrm{mL})$} & \multicolumn{2}{|c|}{ Ln(Syndecan-1) $(\mathrm{pg} / \mathrm{mL})$} \\
\hline & & \multirow{2}{*}{$\begin{array}{l}\text { Result } \\
0.804(0.055)\end{array}$} & \multirow[t]{2}{*}{$\mathrm{p}$-value } & \multirow{2}{*}{$\begin{array}{l}\text { Result } \\
7.04(0.11)\end{array}$} & \multirow[t]{2}{*}{$\mathrm{p}$-value } & \multirow{2}{*}{$\begin{array}{l}\text { Result } \\
10.46(0.14)\end{array}$} & \multirow[t]{2}{*}{ p-value } & \multirow{2}{*}{$\begin{array}{l}\text { Result } \\
8.36(0.09)\end{array}$} & \multirow[t]{2}{*}{ p-value } \\
\hline & & & & & & & & & \\
\hline & Non-septic & $0.422(0.131)$ & $0.021^{\ddagger}$ & $7.14(0.29)$ & $0.943^{\ddagger}$ & $10.29(0.13)$ & $0.860^{\ddagger}$ & $8.68(0.23)$ & $0.292^{\ddagger}$ \\
\hline & Septic & $0.245(0.071)$ & $<0.01^{\stackrel{+}{*}}$ & $7.21(0.19)$ & $0.617^{\ddagger}$ & $11.04(0.15)$ & $0.010^{\ddagger}$ & $8.85(0.09)$ & $0.001^{*}$ \\
\hline \multirow[t]{8}{*}{$\begin{array}{l}\text { Cardiovascular } \\
\text { dysfunction }\end{array}$} & $\begin{array}{l}\text { Present } \\
\text { Absent }\end{array}$ & $\begin{array}{l}0.404(0.067) \\
0.837(0.046)\end{array}$ & $<0.01^{\dagger}$ & $\begin{array}{l}7.29(0.13) \\
6.80(0.10)\end{array}$ & $0.01^{\dagger}$ & $\begin{array}{l}10.84(0.12) \\
10.34(0.17)\end{array}$ & $0.02^{\dagger}$ & $\begin{array}{l}8.79(0.07) \\
8.18(0.10)\end{array}$ & $<0.01^{\dagger}$ \\
\hline & $\begin{array}{l}\text { Lactates } \geq 2 \mathrm{mmol} / \mathrm{L} \\
\text { Lactates }<2 \mathrm{mmol} / \mathrm{L}\end{array}$ & $\begin{array}{l}0.343(0.082) \\
0.750(0.049)\end{array}$ & $<0.01^{\dagger}$ & $\begin{array}{l}7.48(0.23) \\
6.99(0.09)\end{array}$ & $0.030^{\dagger}$ & $\begin{array}{l}10.98(0.18) \\
10.56(0.12)\end{array}$ & $0.071^{\dagger}$ & $\begin{array}{l}9.03(0.09) \\
8.42(0.08)\end{array}$ & $<0.01^{\dagger}$ \\
\hline & $\begin{array}{l}\mathrm{MAP}<65 \mathrm{mmHg} \\
\mathrm{MAP} \geq 65 \mathrm{mmHg}\end{array}$ & $\begin{array}{l}0.445(0.067) \\
0.750(0.066)\end{array}$ & $<0.01^{\dagger}$ & $\begin{array}{l}7.26(0.16) \\
6.97(0.10)\end{array}$ & $0.140^{\dagger}$ & $\begin{array}{l}10.96(0.13) \\
10.37(0.13)\end{array}$ & $<0.01^{\dagger}$ & $\begin{array}{l}8.79(0.09) \\
8.36(0.10)\end{array}$ & $<0.01^{\dagger}$ \\
\hline & $\begin{array}{l}\mathrm{SBP}<90 \mathrm{mmHg} \\
\mathrm{SBP} \geq 90 \mathrm{mmHg}\end{array}$ & $\begin{array}{l}0.493(0.068) \\
0.671(0.070)\end{array}$ & $0.040^{\dagger}$ & $\begin{array}{l}7.25(0.18) \\
7.01(0.10)\end{array}$ & $0.222^{\dagger}$ & $\begin{array}{l}11.01(0.14) \\
10.39(0.12)\end{array}$ & $<0.01^{\dagger}$ & $\begin{array}{l}8.79(0.09) \\
8.40(0.09)\end{array}$ & $<0.01^{\dagger}$ \\
\hline & $\begin{array}{l}\text { With norepinephrine } \\
\text { Without norepineph- } \\
\text { rine } \\
\text { Norepinephrine dose } \\
\text { (mcg } / \mathrm{kg} / \text { minute) }\end{array}$ & $\begin{array}{l}0.475(0.070) \\
0.676(0.068)\end{array}$ & $0.523 \dagger$ & $\begin{array}{l}7.31(0.17) \\
6.94(0.99)\end{array}$ & $0.011 \dagger$ & $\begin{array}{l}10.93(0.14) \\
10.43(0.13)\end{array}$ & $0.325 \dagger$ & $\begin{array}{l}8.76(0.89) \\
8.41(0.09)\end{array}$ & $0.794 \dagger$ \\
\hline & 0.001 to 0.199 & $0.628(0.375)$ & $0.977^{\ddagger}$ & $7.045(0.928)$ & $0.971^{*}$ & $10.807(1.029)$ & $0.458^{\ddagger}$ & $8.535(0.604)$ & $0.882^{\ddagger}$ \\
\hline & 0.200 to 0.499 & $0.529(0.300)$ & $0.666^{\ddagger}$ & $7.193(0.554)$ & $0.723^{*}$ & $11.107(0.757)$ & $0.059^{*}$ & $8.894(0.418)$ & $0.044^{\ddagger}$ \\
\hline & $\geq 0.500$ & $0.265(0.567)$ & $0.010^{\ddagger}$ & $7.637(1.171)$ & $0.025^{\ddagger}$ & $10.874(0.653)$ & $0.262^{\ddagger}$ & $8.824(0.451)$ & $0.073^{\ddagger}$ \\
\hline \multirow[t]{5}{*}{ Renal dysfunction } & $\begin{array}{l}\text { Present } \\
\text { Absent }\end{array}$ & $\begin{array}{l}0.286(0.105) \\
0.700(0.048)\end{array}$ & $<0.01^{\dagger}$ & $\begin{array}{l}7.19(0.21) \\
7.07(0.09)\end{array}$ & $0.566^{\dagger}$ & $\begin{array}{l}10.80(0.17) \\
10.60(0.12)\end{array}$ & $0.339^{\dagger}$ & $\begin{array}{l}8.94(0.11) \\
8.38(0.07)\end{array}$ & $<0.01^{\dagger}$ \\
\hline & AKI severity: & & & & & & & & \\
\hline & Stage 1 & $0.530(0.194)$ & $0.943^{\ddagger}$ & $6.72(0.35)$ & $0.847^{\ddagger}$ & $11.78(0.68)$ & $0.047^{\text {常 }}$ & $8.91(0.30)$ & $0.222^{\ddagger}$ \\
\hline & Stage 2 & $0.262(0.128)$ & $0.009^{\ddagger}$ & $7.35(0.33)$ & $0.819^{\ddagger}$ & $10.52(0.17)$ & $0.994^{\ddagger}$ & $8.86(0.18)$ & $0.102^{\ddagger}$ \\
\hline & Stage 3 & $0.223(0.160)$ & $0.003^{\ddagger}$ & $7.23(0.31)$ & $0.898^{\ddagger}$ & $10.71(0.21)$ & $0.953^{\ddagger}$ & $8.90(0.16)$ & $0.001^{\ddagger}$ \\
\hline $\begin{array}{r}\text { Neurological } \\
\text { dysfunction }\end{array}$ & $\begin{array}{l}\text { Present } \\
\text { Absent }\end{array}$ & $\begin{array}{l}0.556(0.083) \\
0.573(0.070)\end{array}$ & $0.961^{\dagger}$ & $\begin{array}{l}7.00(0.13) \\
7.25(0.14)\end{array}$ & $0.211^{\dagger}$ & $\begin{array}{l}10.79(0.15) \\
10.51(0.12)\end{array}$ & $0.159^{\dagger}$ & $\begin{array}{l}8.56(0.10) \\
8.59(0.10)\end{array}$ & $0.798^{\dagger}$ \\
\hline \multirow[t]{3}{*}{$\begin{array}{l}\text { Respiratory dys- } \\
\text { function }\end{array}$} & $\begin{array}{l}\text { Present } \\
\text { Absent }\end{array}$ & $\begin{array}{l}0.581(0.057) \\
0.557(0.100)\end{array}$ & $0.816^{\dagger}$ & $\begin{array}{l}7.11(0.11) \\
7.12(0.20)\end{array}$ & $0.929^{\dagger}$ & $\begin{array}{l}10.61(0.12) \\
10.79(0.19)\end{array}$ & $0.425^{\dagger}$ & $\begin{array}{l}8.57(0.08) \\
8.57(0.13)\end{array}$ & $0.992^{\dagger}$ \\
\hline & ARDS severity: & & & & & & & & \\
\hline & 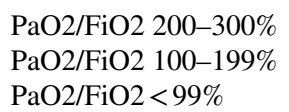 & $\begin{array}{l}0.618(0.082) \\
0.557(0.086) \\
0.558(0.168)\end{array}$ & $\begin{array}{l}0.984^{\ddagger} \\
0.997^{\ddagger} \\
1.000^{\ddagger}\end{array}$ & $\begin{array}{l}7.13(0.18) \\
7.01(0.13) \\
8.22(0.66)\end{array}$ & $\begin{array}{l}0.999^{\ddagger} \\
0.954^{\ddagger} \\
0.249^{\ddagger}\end{array}$ & $\begin{array}{l}10.61(0.22) \\
10.63(0.14) \\
10.29(0.20)\end{array}$ & $\begin{array}{l}0.978^{\ddagger} \\
0.924^{\ddagger} \\
0.892^{\ddagger}\end{array}$ & $\begin{array}{l}8.50(0.12) \\
8.60(0.12) \\
8.87(0.33)\end{array}$ & $\begin{array}{l}0.909^{\ddagger} \\
0.930^{\ddagger} \\
0.848^{\ddagger}\end{array}$ \\
\hline $\begin{array}{l}\text { Hepatic dysfunc- } \\
\text { tion }\end{array}$ & $\begin{array}{l}\text { Present } \\
\text { Absent }\end{array}$ & $\begin{array}{l}0.550(0.119) \\
0.570(0.060)\end{array}$ & $0.868^{\dagger}$ & $\begin{array}{l}7.18(0.19) \\
7.09(0.11)\end{array}$ & $0.668^{\dagger}$ & $\begin{array}{l}10.97(0.18) \\
10.54(0.12)\end{array}$ & $0.047^{\dagger}$ & $\begin{array}{l}8.93(0.12) \\
8.42(0.07)\end{array}$ & $<0.01^{\dagger}$ \\
\hline \multirow[t]{3}{*}{$\begin{array}{l}\text { Hematologic } \\
\text { dysfunction }\end{array}$} & $\begin{array}{l}\text { Present } \\
\text { Absent }\end{array}$ & $\begin{array}{l}0.554(0.071) \\
0.617(0.065)\end{array}$ & $0.939^{\dagger}$ & $\begin{array}{l}7.12(0.13) \\
6.97(0.13)\end{array}$ & $0.257^{\dagger}$ & $\begin{array}{l}10.80(0.12) \\
10.43(0.16)\end{array}$ & $0.078^{\dagger}$ & $\begin{array}{l}8.71(0.09) \\
8.32(0.10)\end{array}$ & $<0.01^{\dagger}$ \\
\hline & $\begin{array}{l}\text { PT time }>12 \mathrm{~s} \\
\text { Normal PT time }\end{array}$ & $\begin{array}{l}0.555(0.071) \\
0.617(0.066)\end{array}$ & $0.539^{\dagger}$ & $\begin{array}{l}7.24(0.17) \\
7.02(0.11)\end{array}$ & $0.299^{\dagger}$ & $\begin{array}{l}10.78(0.12) \\
10.58(0.15)\end{array}$ & $0.319^{\dagger}$ & $\begin{array}{l}8.75(0.09) \\
8.43(0.10)\end{array}$ & $0.024^{\dagger}$ \\
\hline & $\begin{array}{l}\mathrm{PLT} \leq 150 \times 10^{3} / \mathrm{uL} \\
\mathrm{PLT}>150 \times 10^{3} / \mathrm{uL}\end{array}$ & $\begin{array}{l}0.536(0.087) \\
0.618(0.054)\end{array}$ & $0.415^{\dagger}$ & $\begin{array}{l}7.25(0.16) \\
6.98(0.11)\end{array}$ & $0.165^{\dagger}$ & $\begin{array}{l}10.85(0.15) \\
10.48(0.13)\end{array}$ & $0.066^{\dagger}$ & $\begin{array}{l}8.78(0.10) \\
8.37(0.08)\end{array}$ & $<0.01^{\dagger}$ \\
\hline Severity scores & $\begin{array}{l}\text { APACHEII } \\
\text { SAPS2 } \\
\text { SOFA }\end{array}$ & $\begin{array}{l}-0.453 \\
-0.359 \\
-0.314\end{array}$ & $\begin{array}{l}<0.01^{*} \\
<0.01^{*} \\
<0.01^{*}\end{array}$ & $\begin{array}{l}0.039 \\
0.083 \\
0.218\end{array}$ & $\begin{array}{l}0.752^{*} \\
0.501^{*} \\
0.074^{*}\end{array}$ & $\begin{array}{l}0.226 \\
0.229 \\
0.335\end{array}$ & $\begin{array}{l}0.063^{*} \\
0.060^{*} \\
<0.01^{*}\end{array}$ & $\begin{array}{l}0.340^{*} \\
0.366^{*} \\
0.553^{*}\end{array}$ & $\begin{array}{l}<0.01 \\
<0.01 \\
<0.01\end{array}$ \\
\hline
\end{tabular}

Results are presented as mean (standard deviation) or Pearsons' $r$

APACHEII Acute physiology and chronic health evaluation II, ARDS acute respiratory distress syndrome, $C V$ cardiovascular, $L n$ natural logarithm, MAP mean arterial pressure, $P L T$ platelets, $P T$ prothrombin time, SBP systolic blood pressure, SAPSII simplified acute physiology score II, SOFA sequential organ failure assessment

${ }^{\dagger}$ Bivariate variables were compared using Students t-test

†Variables with more than 2 categories were compared with Dunnetts' test, presented p-values refer to the absence of condition (control)

*For comparisons between continuous variables, the Pearsons' correlation test was used 
$\mathrm{Ln}(\mathrm{RHI})$ was significantly lower in patients with circulatory shock $(\mathrm{p}<0.001)$, and cardiovascular dysfunction $(\mathrm{p}<0.01)$, including all 3 variables used as criteria (lactates, mean arterial pressure (MAP) and systolic blood pressure (SBP); p < 0.01) (Fig. 3a and b). The association with lactate levels remained significant $(\mathrm{B}-0.365 ; \mathrm{p}<0.001 ; 95 \%$ $\mathrm{CI}-0.132$ to -0.042 ) after adjusting for MAP (B 0.158; $95 \% \mathrm{CI}-0.003$ to 0.013 ; SBP (B $0.092 ; 95 \% \mathrm{CI}-0.003$ to $0.007)$ and norepinephrine dose $(-0.154 ; 95 \%$ CI -0.141 to 0.017 ) in a multivariate linear regression model. Although there was a tendency toward lower $\ln (\mathrm{RHI})$ in septic shock, compared to non-septic shock, the difference was not significant $(\mathrm{p}=0.369)$.

Lower $\ln (\mathrm{RHI})$ was found in renal dysfunction $(\mathrm{p}<0.01)$ in proportion to severity of acute kidney injury $(\mathrm{p}<0.01)$, serum creatinine (Pearson's $\mathrm{r}-0.164 ; \mathrm{p}=0.01$ ) and daily urinary volume (Pearson's r $0.390 ; p<0.01$ ) (Fig. 3c). This association was independent of age, sepsis, comorbidities such as chronic kidney disease, or macrovascular determinants such as arterial pressure or the need for vasopressors (B - 2.359, $\mathrm{p}=0.01$, OR $0.094,95 \%$ CI $0.024-0.370$ ). The association between other endothelial biomarkers and organic dysfunctions are found in Table 4. In a multivariate analysis to verify if the associations between the biomarkers and organic dysfunctions were influenced by the presence of sepsis and other comorbidities, $\ln (\mathrm{RHI})$ was independently associated with cardiovascular $(B-2.739 p=0.001$, OR 0.065, 95\% CI 0.016-0.259) and renal dysfunctions (B $-2.359, \mathrm{p}=0.001$, OR $0.094,95 \%$ CI 0.024-0.370). Syndecan-1 was also independently associated with cardiovascular (B 2.559, $\mathrm{p}=0.001$, OR 12.917, 95\% CI 3.323-50.216) and renal dysfunctions (B 2.258, $\mathrm{p}=0.001$, OR $9.566,95 \% \mathrm{CI}$ 2.700-33.895). Endocan and sE-selectin prediction of cardiovascular and renal dysfunctions were highly dependent on the presence of sepsis.

\section{Discussion}

The objective of this study was to investigate an association between RH-PAT and outcomes in the critically ill patient, that could suggest this method of evaluating endothelial function as a tool for assessing prognosis in the ICU. Until recently, data on RH-PAT in the critically ill patient was lacking. Davis et al. measured RHI on admission and after 2-4 days in septic patients and found a significant increase that correlated with increasing MAP and decreasing inflammatory markers [10]. In another study with a similar design, Nobre et al. also found a significant association between sepsis severity and RH-PAT [25]. However, none of the studies found an association between RH-PAT and mortality, and the authors claimed that the low mortality in both cohorts may have underpowered the results. In non-septic patients,
Bergström et al. found RH-PAT to decrease from admission to day 3 after subarachnoid haemorrhage and identified a significant correlation with mortality [26]. Furthermore, other methods of accessing reactive hyperaemia such as FMD and peak hyperaemic flow velocity in the brachial artery found promising results regarding the association with mortality in the septic and non-septic critically ill patients admitted to the ICU [27-29].

Contrarily to the results by Davis et al. and Nobre et al., our results suggest that RH-PAT may be an independent predictor of early mortality, and lower $\ln (\mathrm{RHI})$ values are associated with higher mortality even after adjusting for confounding factors [10, 25]. The improvement in RH-PAT over the disease course in survivors suggests that RH-PAT is acutely impaired in critical illness and that endothelial dysfunction may be reversible. These results have not been described before and add to the relevance of RH-PAT as a useful tool to stratify severity in the ICU. The concept of an acute RH-PAT impairment is further supported by studies demonstrating that RH-PAT decreases from basal values in patients submitted to cardiopulmonary bypass with continuous flow or catheterization of the radial artery [30, 31].

RH-PAT was also correlated with disease severity when measured by APACHE-II, SAPS-II and SOFA scores, which is supported by the findings of other authors in sepsis and septic shock [10, 25], dengue fever [32], malaria [33, 34] and ST-elevation myocardial infarctions [14]. Vasopressors such as epinephrine and norepinephrine are widely used in critical care to treat life-threatening hypotension, especially in patients who do not respond to fluid resuscitation. Norepinephrine is usually titrated to maintain a MAP that allows an adequate systemic blood perfusion. As norepinephrine binds mainly to alpha-1 adrenergic receptors of vascular smooth muscle and induces vasoconstriction, vascular reactivity testing, such as RH-PAT, is expected to be impaired by the presence of norepinephrine in supraphysiological levels. However, the findings that $\ln (\mathrm{RHI})$ correlated negatively with lactate levels and acute kidney injury, irrespectively of mean arterial pressure (MAP) or systolic blood pressure (SBP), and after adjusting for vasopressor dose, suggests that RH-PAT is mainly associated with microvascular dysfunction, that it may be independent of macrovascular parameters and that norepinephrine may not significantly impair RHPAT. Our findings are further supported by the research by Van Ierssel et al. who also found RH-PAT to be significantly compromised in critically ill patients and independent of vasopressor dose or the need for vasopressors at the time of evaluation [35].

Although the physiopathology behind microvascular dysfunction is complex and specific between organs and types of shock, evidence suggests that low levels of endothelial NO synthase (eNOS) activity are common and partially responsible for the perfusion mismatches in many organs, 
including the kidneys [36-39]. It is also well recognized that a subset of patients with AKI still experience progressive renal abnormalities despite adequate circulatory parameters, suggesting that hypoperfusion is not the only cause for renal dysfunction [39, 40]. AS RH-PAT is partially dependent on eNOS activity and reflects glycocalyx integrity in acute diseases, we believe it may be helpful to identify patients with microvascular dysfunction, which is also eNOS dependent, in whom hemodynamical parameters are still stable [41].

RH-PAT and Syndecan-1, a transmembrane heparan sulphate proteoglycan detached from the endothelium in the presence of glycocalyx degradation, were both consistently associated with mortality, cardiovascular and renal dysfunctions, even after adjusting for comorbidities, age and the presence of sepsis, which is unsurprising, as we previously demonstrated that RH-PAT is highly correlated with glycocalyx degradation and Syndecam-1 serum levels [24, 42]. Endocan is a proteoglycan mainly, but not exclusively, expressed by pulmonary and renal endothelial cells, that is secreted in response to inflammatory conditions in which the endothelium is impaired such as acute lung injury in trauma patients and pneumonia [43]. In our study we did not find any association with mortality, organic dysfunctions, even after adjusting for the presence of sepsis or source of the disease, which makes this biomarker less promising than RHPAT. Soluble E-selectin, an endothelial adhesion molecule secreted in a wide array of inflammatory conditions, was associated with cardiovascular, renal and haematological dysfunction in our study [44]. However, this difference was not as consistent after adjusting for the presence of sepsis, and sE-selectin did not seem a reliable marker of organic dysfunction outside septic ICU patients.

Although we controlled our results for most major known factors influencing mortality and RH-PAT, we cannot exclude minor influences from unmeasured comorbidities such as thyroid or adrenal function, and unmeasured environmental factors such luminosity and noise. Furthermore, we cannot ascertain if medical interventions such as drug perfusion, other than vasopressors, and overall stress induced by the admission to the ICU may have influenced RH-PAT and contributed to the changes verified over time in survivors. A bias was introduced when estimating mortality as patients with more severe disease died in the first $24 \mathrm{~h}$ and a significant number of patients were discharged before the 2nd RH-PAT measurement, which may have underpowered our results.

The unexpected lack of association between RH-PAT and organic dysfunctions other than cardiovascular and renal may be explained by a mismatch between the used definitions and the physiopathology behind the dysfunction. Hughes et al. described an independent association between RH-PAT and the duration of sepsis-induced delirium and coma [45]. We hypothesize that the Glasgow Coma Scale, may not be the best instrument for measuring neurological impairment in the critically ill patient as it is static and subject to interobserver variability. Evidence that the Acute Respiratory Distress Syndrome (ARDS) is associated with peripheral RH comes from Orbegozo-Cortés et al. who proved, using near-infrared spectroscopy after a vascular occlusion test in the forearm, that microvascular reactivity is altered early in ARDS, and the changes are directly related to the severity of the disease [46]. The same rationale may be used for hepatocellular and haematologic dysfunctions, both directly influenced by eNOS activity, in which other parameters may be more sensitive than total bilirubin or platelet number [47-50]. Hence, we suggest that currently available clinical variables may not reflect the full extent of organ dysfunction nor the pathogenesis behind it.

As endothelial dysfunction may be the primum movens behind acute organic dysfunction and consequent mortality, finding a clinically useful biomarker that reflects acute systemic endothelial dysfunction and simplifies such complex process will not only provide new therapeutic targets, but also remodel critical illness from a biological level in which organ support is the main target, to a fully treatable disease. Nonetheless, as the endothelium is phenotypically different between vascular beds and mechanisms behind RH may differ, RH-PAT may not reflect the full extent of endothelial dysfunction as it does not identify organs in which endothelial dysfunction manifests in NO-independent mechanisms [7, 51-53].

\section{Conclusion}

Recognizing RH-PAT as a window into eNOS activity and characterizing organic functions in which eNOS is essential, may turn RH-PAT into a valuable tool for the identification of conditions in which specific eNOS-directed therapeutics may be beneficial. Despite limitations, the present results add to a growing corpus of evidence that RH-PAT acts as a promising predictor of disease severity, early mortality and cardiovascular and renal dysfunction in the critically ill. To our knowledge, this is the first report suggesting that RHPAT kinetics during disease may also be relevant. Future studies should aim to replicate results on a larger scale to further validate our results.

Acknowledgements We would like to acknowledge Professor Ben Sweeney for providing writing support in English.

Author contributions All authors contributed to the study conception and design. LM, LS and AS clinically managed the patients. LM, MVS, RG and LS designed the study and prepared the material. LM and LS collected, analysed and interpreted the patient data and results. RG analysed the data and contributed with statistical support. SM provided laboratory and writing support. The first draft of the manuscript was 
written by LM and all authors commented on previous versions of the manuscript. All authors read and approved the final manuscript.

Funding Professor Rita Gaio was partially supported by CMUP (UID/ MAT/00144/2013), which is funded by FCT (Portugal) with national (MEC) and European structural funds (FEDER), under the partnership agreement PT2020.

Data availability The datasets used and/or analysed during the current study are available from the corresponding author on reasonable request.

\section{Compliance with ethical standards}

Conflict of interest The authors declare they have no conflict of interests.

Ethical approval This study was approved by the local ethics committee (Centro Hospitalar de São João, ref 205/11) and has therefore been performed in accordance with the ethical standards laid down in the 1964 Declaration of Helsinki and its later amendments.

Informed consent Written consent was obtained from the patient, if awake, or from the legal representative. Patients signed informed consent regarding publishing their data.

\section{References}

1. Resche-Rigon M, Azoulay E, Chevret S. Evaluating mortality in intensive care units: contribution of competing risks analyses. Crit Care. 2006;10(1):R5. https://doi.org/10.1186/cc3921.

2. Halpern NA, Pastores SM. Critical care medicine in the United States 2000-2005: an analysis of bed numbers, occupancy rates, payer mix, and costs. Crit Care Med. 2010;38(1):65-71. https:// doi.org/10.1097/CCM.0b013e3181b090d0.

3. Mukhopadhyay A, Tai BC, See KC, Ng WY, Lim TK, Onsiong S, Ee S, Chua MJ, Lee PR, Loh ML, et al. Risk factors for hospital and long-term mortality of critically ill elderly patients admitted to an intensive care unit. Biomed Res Int. 2014;2014:960575. https://doi.org/10.1155/2014/960575.

4. Aird WC. Spatial and temporal dynamics of the endothelium. J Thromb Haemost. 2005;3(7):1392-406. https://doi.org/10.111 1/j.1538-7836.2005.01328.x.

5. Ait-Oufella H, Maury E, Lehoux S, Guidet B, Offenstadt G. The endothelium: physiological functions and role in microcirculatory failure during severe sepsis. Intensive Care Med. 2010;36(8):1286-98. https://doi.org/10.1007/s00134-010-1893-6.

6. De Backer D, Creteur J, Dubois MJ, Sakr Y, Vincent JL. Microvascular alterations in patients with acute severe heart failure and cardiogenic shock. Am Heart J. 2004;147(1):91-9. https://doi. org/10.1016/j.ahj.2003.07.006.

7. Aird WC. Phenotypic heterogeneity of the endothelium: I. Structure, function, and mechanisms. Circ Res. 2007;100(2):158-73. https://doi.org/10.1161/01.RES.0000255691.76142.4a.

8. Raff U, Ott C, John S, Schmidt BM, Fleischmann EH, Schmieder RE. Nitric oxide and reactive hyperemia: role of location and duration of ischemia. Am J Hypertens. 2010;23(8):865-9. https ://doi.org/10.1038/ajh.2010.72.

9. Kuvin JT, Mammen A, Mooney P, Alsheikh-Ali AA, Karas RH. Assessment of peripheral vascular endothelial function in the ambulatory setting. Vasc Med. 2007;12(1):13-6. https://doi. org/10.1177/1358863X06076227.
10. Davis JS, Yeo TW, Thomas JH, McMillan M, Darcy CJ, McNeil YR, Cheng AC, Celermajer DS, Stephens DP, Anstey NM. Sepsis-associated microvascular dysfunction measured by peripheral arterial tonometry: an observational study. Crit Care. 2009;13(5):R155. https://doi.org/10.1186/cc8055.

11. Bonetti PO, Pumper GM, Higano ST, Holmes DR Jr, Kuvin JT, Lerman A. Noninvasive identification of patients with early coronary atherosclerosis by assessment of digital reactive hyperemia. J Am Coll Cardiol. 2004;44(11):2137-41. https:// doi.org/10.1016/j.jacc.2004.08.062.

12. Duffy MJ, Mullan BA, Craig TR, Shyamsundar M, MacSweeney RE, Thompson G, Stevenson M, McAuley DF. Impaired endothelium-dependent vasodilatation is a novel predictor of mortality in intensive care. Crit Care Med. 2011;39(4):629-35. https://doi.org/10.1097/CCM.0b013e318206bc4a.

13. Matsuzawa Y, Kwon TG, Lennon RJ, Lerman LO, Lerman A. Prognostic value of flow-mediated vasodilation in brachial artery and fingertip artery for cardiovascular events: a systematic review and meta-analysis. J Am Heart Assoc. 2015. https ://doi.org/10.1161/JAHA.115.002270.

14. Bravo Baptista S, Faustino M, Brizida L, Loureiro J, Augusto J, Abecasis J, Monteiro C, Leal P, Nedio M, Farto EAP, et al. Early peripheral endothelial dysfunction predicts myocardial infarct extension and microvascular obstruction in patients with ST-elevation myocardial infarction. Rev Port Cardiol. 2017;36(10):731-42. https://doi.org/10.1016/j. repc.2017.01.006.

15. Singer M, Deutschman CS, Seymour CW, Shankar-Hari M, Annane D, Bauer M, Bellomo R, Bernard GR, Chiche JD, Coopersmith $\mathrm{CM}$, et al. The third international consensus definitions for sepsis and septic shock (sepsis-3). JAMA. 2016;315(8):801-10. https://doi.org/10.1001/jama.2016.0287.

16. Rhodes A, Evans LE, Alhazzani W, Levy MM, Antonelli M, Ferrer R, Kumar A, Sevransky JE, Sprung CL, Nunnally ME, et al. Surviving sepsis campaign: international guidelines for management of sepsis and septic shock: 2016. Intensive Care Med. 2017;43(3):304-77. https://doi.org/10.1007/s00134-017-4683-6.

17. Cecconi M, De Backer D, Antonelli M, Beale R, Bakker J, Hofer C, Jaeschke R, Mebazaa A, Pinsky MR, Teboul JL, et al. Consensus on circulatory shock and hemodynamic monitoring. Task force of the European Society of Intensive Care Medicine. Intensive Care Med. 2014;40(12):1795-815. https://doi.org/10.1007/s0013 4-014-3525-Z

18. Minne L, Abu-Hanna A, de Jonge E. Evaluation of SOFA-based models for predicting mortality in the ICU: a systematic review. Crit Care. 2008;12(6):R161. https://doi.org/10.1186/cc7160.

19. Force ADT, Ranieri VM, Rubenfeld GD, Thompson BT, Ferguson ND, Caldwell E, Fan E, Camporota L, Slutsky AS. Acute respiratory distress syndrome: the Berlin definition. JAMA. 2012;307(23):2526-33. https://doi.org/10.1001/jama.2012.5669.

20. Group TKDIGOKW. Definition and classification of acute kidney injury. Kidney Int Suppl. 2012;2:19-36.

21. Axtell AL, Gomari FA, Cooke JP. Assessing endothelial vasodilator function with the Endo-PAT 2000. J Vis Exp. 2010. https://doi. org/10.3791/2167.

22. Moerland M, Kales AJ, Schrier L, van Dongen MG, Bradnock D, Burggraaf J. Evaluation of the EndoPAT as a tool to assess endothelial function. Int J Vasc Med. 2012;2012:904141. https:// doi.org/10.1155/2012/904141.

23. Firth D. Bias reduction of maximum likelihood estimates. Biometrika. 1993;80(1):27-38.

24. Malheiro L GR, Vaz da Silva M, Martins S, Sampaio S, QuelhasSantos J, Cerqueira A, Sarmento A, Santos L. (2020) Reactive hyperemia correlates with the presence of sepsis and glycocalyx degradation in the intensive care unit: a prospective cohort study. Revista Brasileira de Terapia Intensiva. 32:in press. 
25. Nobre V, Ataide TB, Brant LC, Oliveira CR, Rodrigues LV, Ribeiro AL, Lopes FB, Saraiva IE, Andrade MV. Use of reactive hyperemia-peripheral arterial tonometry and circulating biological markers to predict outcomes in sepsis. Rev Bras Ter Intensiva. 2016;28(4):387-96. https://doi.org/10.5935/0103507X.20160072.

26. Bergstrom A, Staalso JM, Romner B, Olsen NV. Impaired endothelial function after aneurysmal subarachnoid haemorrhage correlates with arginine:asymmetric dimethylarginine ratio. Br J Anaesth. 2014;112(2):311-8. https://doi.org/10.1093/ bja/aet331.

27. Becker L, Prado K, Foppa M, Martinelli N, Aguiar C, Furian T, Clausell N, Rohde LE. Endothelial dysfunction assessed by brachial artery ultrasound in severe sepsis and septic shock. J Crit Care. 2012;27(3):316. https://doi.org/10.1016/j. jerc.2011.08.002.

28. Wexler O, Morgan MA, Gough MS, Steinmetz SD, Mack CM, Darling DC, Doolin KP, Apostolakos MJ, Graves BT, Frampton MW, et al. Brachial artery reactivity in patients with severe sepsis: an observational study. Crit Care. 2012;16(2):R38. https ://doi.org/10.1186/cc11223.

29. Bonjorno Junior JC, Caruso FR, Mendes RG, da Silva TR, Biazon T, Rangel F, Phillips SA, Arena R, Borghi-Silva A. Noninvasive measurements of hemodynamic, autonomic and endothelial function as predictors of mortality in sepsis: a prospective cohort study. PLoS One. 2019;14(3):e0213239. https ://doi.org/10.1371/journal.pone.0213239.

30. Sangalli F, Guazzi M, Senni S, Sala W, Caruso R, Costa MC, Formica F, Avalli L, Fumagalli R. Assessing endothelial responsiveness after cardiopulmonary bypass: insights on different perfusion modalities. J Cardiothorac Vasc Anesth. 2015;29(4):912-6. https://doi.org/10.1053/j.jvca.2014.11.008.

31. Antonopoulos AS, Latsios G, Oikonomou E, Aznaouridis K, Papanikolaou A, Syrseloudis D, Siasos G, Vavuranakis M, Toutouzas K, Tousoulis D. Long-term endothelial dysfunction after trans-radial catheterization: a meta-analytic approach. J Card Surg. 2017;32(8):464-73. https://doi.org/10.1111/jocs.13181.

32. Yacoub S, Lam PK, Huynh TT, Nguyen Ho HH, Dong Thi HT, Van NT, Lien LT, Ha QNT, Le DHT, Mongkolspaya J, et al. Endothelial nitric oxide pathways in the pathophysiology of dengue: a prospective observational study. Clin Infect Dis. 2017;65(9):1453-61. https://doi.org/10.1093/cid/cix567.

33. Barber BE, William T, Grigg MJ, Piera KA, Chen Y, Wang $\mathrm{H}$, Weinberg JB, Yeo TW, Anstey NM. Nitric oxide-dependent endothelial dysfunction and reduced arginine bioavailability in plasmodium vivax malaria but no greater increase in intravascular hemolysis in severe disease. J Infect Dis. 2016;214(10):1557-644. https://doi.org/10.1093/infdis/jiw427.

34. Yeo TW, Lampah DA, Gitawati R, Tjitra E, Kenangalem E, McNeil YR, Darcy CJ, Granger DL, Weinberg JB, Lopansri BK, et al. Impaired nitric oxide bioavailability and L-arginine reversible endothelial dysfunction in adults with falciparum malaria. J Exp Med. 2007;204(11):2693-704. https://doi.org/10.1084/ jem.20070819.

35. van Ierssel SH, Van Craenenbroeck EM, Hoymans VY, Vrints CJ, Conraads VM, Jorens PG. Endothelium dependent vasomotion and in vitro markers of endothelial repair in patients with severe sepsis: an observational study. PLoS One. 2013;8(8):e69499. https://doi.org/10.1371/journal.pone.00694 99.

36. Engelberger RP, Pittet YK, Henry H, Delodder F, Hayoz D, Chiolero RL, Waeber B, Liaudet L, Berger MM, Feihl F. Acute endotoxemia inhibits microvascular nitric oxide-dependent vasodilation in humans. Shock. 2011;35(1):28-34. https://doi. org/10.1097/SHK.0b013e3181ec71ab.
37. Aldamiz-Echevarria L, Andrade F. Asymmetric dimethylarginine, endothelial dysfunction and renal disease. Int J Mol Sci. 2012;13(9):11288-311. https://doi.org/10.3390/ijms130911288.

38. Padberg JS, Wiesinger A, di Marco GS, Reuter S, Grabner A, Kentrup D, Lukasz A, Oberleithner H, Pavenstadt H, Brand $\mathrm{M}$, et al. Damage of the endothelial glycocalyx in chronic kidney disease. Atherosclerosis. 2014;234(2):335-43. https://doi. org/10.1016/j.atherosclerosis.2014.03.016.

39. Post EH, Kellum JA, Bellomo R, Vincent JL. Renal perfusion in sepsis: from macro- to microcirculation. Kidney Int. 2017;91(1):45-60. https://doi.org/10.1016/j.kint.2016.07.032.

40. Albanese J, Leone M, Garnier F, Bourgoin A, Antonini F, Martin C. Renal effects of norepinephrine in septic and nonseptic patients. Chest. 2004;126(2):534-9. https://doi.org/10.1378/ chest.126.2.534.

41. Hedetoft M, Olsen NV. Evaluation of endothelial function by peripheral arterial tonometry and relation with the nitric oxide pathway. Nitric Oxide. 2014;42:1-8. https://doi.org/10.1016/j. niox.2014.07.003.

42. Nelson A, Johansson J, Tyden J, Bodelsson M. Circulating syndecans during critical illness. APMIS. 2017;125(5):468-75. https://doi.org/10.1111/apm.12662.

43. Kechagia M, Papassotiriou I, Gourgoulianis KI. Endocan and the respiratory system: a review. Int J Chron Obstruct Pulmon Dis. 2016;11:3179-87. https://doi.org/10.2147/COPD.S118692.

44. Cummings CJ, Sessler CN, Beall LD, Fisher BJ, Best AM, Fowler AA 3rd. Soluble E-selectin levels in sepsis and critical illness. Correlation with infection and hemodynamic dysfunction. Am J Respir Crit Care Med. 1997;156(2 Pt 1):431-7. https ://doi.org/10.1164/ajrccm.156.2.9509017.

45. Hughes CG, Morandi A, Girard TD, Riedel B, Thompson JL, Shintani AK, Pun BT, Ely EW, Pandharipande PP. Association between endothelial dysfunction and acute brain dysfunction during critical illness. Anesthesiology. 2013;118(3):631-9. https://doi.org/10.1097/ALN.0b013e31827bd193.

46. Orbegozo Cortes D, Rahmania L, Irazabal M, Santacruz C, Fontana V, De Backer D, Creteur J, Vincent JL. Microvascular reactivity is altered early in patients with acute respiratory distress syndrome. Respir Res. 2016;17(1):59. https://doi.org/10.1186/ s12931-016-0375-y.

47. Kortgen A, Paxian M, Werth M, Recknagel P, Rauchfuss F, Lupp A, Krenn CG, Muller D, Claus RA, Reinhart K, et al. Prospective assessment of hepatic function and mechanisms of dysfunction in the critically ill. Shock. 2009;32(4):358-65. https ://doi.org/10.1097/SHK.0b013e31819d8204.

48. Dewitte A, Lepreux S, Villeneuve J, Rigothier C, Combe C, Ouattara A, Ripoche J. Blood platelets and sepsis pathophysiology: a new therapeutic prospect in critical ill patients? Ann Intensive Care. 2017;7(1):115. https://doi.org/10.1186/s1361 3-017-0337-7.

49. Bor-Kucukatay M, Wenby RB, Meiselman HJ, Baskurt OK. Effects of nitric oxide on red blood cell deformability. Am J Physiol Heart Circ Physiol. 2003;284(5):H1577-1584. https:// doi.org/10.1152/ajpheart.00665.2002.

50. Iwakiri Y, Kim MY. Nitric oxide in liver diseases. Trends Pharmacol Sci. 2015;36(8):524-36. https://doi.org/10.1016/j. tips.2015.05.001.

51. Aird WC. Phenotypic heterogeneity of the endothelium: II. Representative vascular beds. Circ Res. 2007;100(2):174-90. https ://doi.org/10.1161/01.RES.0000255690.03436.ae.

52. Hamburg NM, Palmisano J, Larson MG, Sullivan LM, Lehman BT, Vasan RS, Levy D, Mitchell GF, Vita JA, Benjamin EJ. Relation of brachial and digital measures of vascular function in the community: the Framingham heart study. Hypertension. 
2011;57(3):390-6. https://doi.org/10.1161/HYPERTENSI ONAHA.110.160812.

53. Wong BJ, Wilkins BW, Holowatz LA. Minson CT (2003) Nitric oxide synthase inhibition does not alter the reactive hyperemic response in the cutaneous circulation. J Appl Physiol. 1985;95(2):504-10. https://doi.org/10.1152/japplphysiol.00254 .2003 .
Publisher's Note Springer Nature remains neutral with regard to jurisdictional claims in published maps and institutional affiliations. 